\title{
INCREMENTO NO DESENVOLVIMENTO DO PORTA-ENXERTO DE PESSEGUEIRO ‘Aldrighi’ POR FUNGOS MICORRÍZICOS ARBUSCULARES AUTÓCTONES
}

\author{
Development increase of 'Aldrighi' peach rootstocks by indigenous arbuscular mycorrhizal fungi
}

\author{
José Luis da Silva Nunes¹, Paulo Vitor Dutra de Souza², Gilmar Arduino Bettio Marodin², José Carlos Fachinello ${ }^{3}$
}

RESUMO

Objetivou-se, neste trabalho, avaliar a influência de três espécies de fungos micorrízicos arbusculares (FMA) isolados de pomares de pessegueiro sobre o crescimento vegetativo, nutrição mineral e substâncias de reserva em plantas do porta-enxerto de pessegueiro cv. Aldrighi [Prunus persica (L.) Batsch]. O delineamento experimental utilizado foi o de blocos casualizados, com dez plantas por parcela e quatro repetições. As plantas inoculadas com Glomus etunicatum apresentaram maior altura, diâmetro, área foliar, biomassa fresca e seca, nutrição mineral e substâncias de reserva da parte aérea, enquanto as inoculadas com Glomus clarum induziram um crescimento intermediário, superior àquelas inoculadas com Gigaspora margarita, que apresentaram resultados semelhantes às plantas não inoculadas. $\mathrm{O}$ desempenho foi relacionado com as taxas de colonização que nas plantas inoculadas com Glomus etunicatum e Glomus clarum foram de $92 \%$ e $77 \%$ respectivamente, enquanto Gigaspora margarita colonizou somente $30 \%$ das raízes.

Termos para indexação: Prunus persica, endomicorrizas, propagação, fisiologia.

\section{ABSTRACT}

This work aimed to evaluate the influence of three arbuscular mycorrhizal fungi (AMF) species on the vegetative growth, mineral nutrition and carbohidrate contents on peach rootstocks cv. Aldrighi [Prunus persica (L.) Batsch]. The experimental desing was the one of randomized blocks, with ten plants per plots and four repetitions. Plants inoculated with Glomus etunicatum presented larger stem height, stem diameter, foliar area, fresh and dry shoot biomass, leaf mineral nutrition and carbohidrate contents, while those inoculated with Glomus clarum induced an intermediate growth, higher to those inoculated with Gigaspora margarita that presented results similar to the non inoculated plants. Plant growth performance was related to colonization taxes, which were, respectively, 92\%, 77\% and 30\% to Glomus etunicatum, Glomus clarum and Gigaspora margarita inoculated plants.

Index terms: Prunus persica, endomycorrhizae, plant propagation, plant physiology.

(Recebido em 14 de março de 2008 e aprovado em 11 de julho de 2008)

\section{INTRODUÇÃO}

Os fungos formadores de micorrizas arbusculares são componentes comuns da rizosfera e das raízes da maioria das plantas superiores. Essa simbiose, que é do tipo biotrófico mutualística, está amplamente distribuída no reino vegetal (DODD, 2000). Os benefícios dessa simbiose, expressos principalmente como estímulo ao crescimento vegetal, devem-se a fatores nutricionais, principalmente ao aumento da absorção de nitrogênio (AGOSTINI, 2002; SOUZA, 2000), fósforo (SOUZA, 2000) e potássio (SILVEIRA et al., 2002).

A interpretação correta da dinâmica local de espécies de microrganismos presentes em uma área é fundamental para se entender a influência dos FMA sobre o equilíbrio das plantas estabelecidas nesse local (RILLING et al., 2002). Nesse aspecto, as espécies de FMA autóctones de uma determinada região, que já são adaptadas às condições edafoclimáticas da mesma, tendem a conferir um maior potencial de resposta, quando inoculadas nas plantas a serem cultivadas nessa região, ainda que seja variável segundo a espécie do endófito (SILVEIRA et al., 2002). Com isso, o uso de espécies de fungos micorrízicos arbusculares nativas de pomares de pessegueiro tende a ser uma boa opção a ser utilizada na produção de mudas dessa cultura.

Objetivou-se, neste trabalho, avaliar a influência de diferentes espécies de FMA nativas de pomares de

\footnotetext{
'Engenheiro Agrônomo, Doutor em Fitotecnia - Departamento de Horticultura e Silvicultura/DHS - Faculdade de Agronomia - Universidade Federal do Rio Grande do Sul/UFRGS - Rua Sílvio Silveira Soares, 2406/130 - Camaquã - 91910-460 - Porto Alegre, RS - silva.nunes@ufrgs.br

²Engenheiros Agrônomos, Doutores em Agronomia, Professores Adjunto - Departamento de Horticultura e Silvicultura/DHS - Faculdade de Agronomia Universidade Federal do Rio Grande do Sul/UFRGS - Avenida Bento Gonçalves, 7712 - Agonomia - Cx. P. 15100 - $91540-000$ - Porto Alegre, RS pvdsouza@ufrgs.br; marodin@ufrgs.br

${ }^{3}$ Engenheiro Agrônomo, Doutor em Agronomia, Professor Titular - Departamento de Fitotecnia/DF - Faculdade de Agronomia Eliseu Maciel/FAEM Universidade Federal de Pelotas/UFPel - Cx. P. 354 - 96010-900 - Pelotas, RS - jfachi@ufpel.tche.br
} 
pessegueiro sobre o desenvolvimento vegetativo e teores de nutrientes e substâncias de reservas da parte aérea de plantas do porta-enxerto de pessegueiro cv. Aldrighi.

\section{MATERIALE MÉTODOS}

Caroços do porta-enxerto cv. Aldrighi foram estratificados em recipientes (caixa plástica de $40 \mathrm{~cm} \mathrm{X}$ $28 \mathrm{~cm}$ X $10 \mathrm{~cm}$ ) contendo areia e colocados, por um período de 45 dias, em refrigerador à temperatura de $4^{\circ} \mathrm{C}$, visando interromper a dormência do embrião e facilitar a germinação. A areia foi previamente autoclavada a $120^{\circ} \mathrm{C}$ por uma hora. Essa operação foi realizada no laboratório do Departamento de Horticultura e Silvicultura, da Faculdade de Agronomia da Universidade Federal do Rio Grande do Sul (UFRGS).

Logo após o período de estratificação, as sementes foram retiradas dos caroços e semeadas em caixas plásticas $(40 \mathrm{~cm} \mathrm{X} 28 \mathrm{~cm} \mathrm{X} 10 \mathrm{~cm})$, preenchidas com areia desinfestada e mantidas em casa de nebulização. Quando as plântulas apresentava cerca de $5 \mathrm{~cm}$ de altura da parte aérea, foram repicadas para sacos plásticos pretos (5 litros), contendo substrato constituído de terra oriunda de um solo Argissolo Vermelho distrófico típico (EMBRAPA, 1999), areia (granulometria média, entre 0,6 e $1 \mathrm{~mm}$ ) e resíduo decomposto de casca de acácia negra (1:1:1, v:v.v). O substrato foi previamente desinfestado com solução de formaldeído, a $10 \%$.

Imediatamente antes da repicagem procedeu-se à adição dos inóculos de FMA ao substrato (30 gramas por saco plástico, aproximadamente 10 esporos por grama de inóculo), em uma camada situada na altura intermediária de cada recipiente (aproximadamente a $15 \mathrm{~cm}$ de altura). $\mathrm{O}$ inóculo era constituído de raízes e solo rizósférico de braquiária (Brachiaria decumbens Stapf.), espécie vegetal utilizada para multiplicar, através de cultivo monospórico, cada uma das espécies de FMA. Os esporos foram isolados a partir de amostras de solo coletadas em pomares de pessegueiro, através do método de lavagem, decantação, peneiramento (GERDEMANN \& NICOLSON, 1963) e centrifugação (JENKINS, 1964). A identificação morfológica das espécies foi realizada através da observação em microscópio óptico, segundo Schenck \& Perez (1988). As espécies de FMA testadas foram Gigaspora margarita (BECKER \& HALL), Glomus clarum (NICOL. \& SCHENCK) e Glomus etunicatum (BECKER \& GERD), além das testemunhas, que não receberam inóculo. $\mathrm{O}$ delineamento experimental utilizado foi o de blocos casualizados, com quatro tratamentos, quatro repetições por tratamento e 10 plantas por repetição.

O experimento foi conduzido em casa de vegetação equipada com sistema de irrigação por aspersão, localizada no setor de Horticultura da Estação Experimental Agronômica (EEA) da UFRGS, Km 146 da BR 290, município de Eldorado do Sul (RS), tendo sido iniciado em 02 de setembro de 2005.

Onze meses após a semeadura, mediu-se a altura e o diâmetro do colo das plantas sendo, em seguida, colhidas para determinação da biomassa fresca e seca da parte aérea (folhas e hastes), área foliar, através do uso de medidor de área foliar marca Li-Cor (modelo LI - 3000), teores de nutrientes (folhas e hastes), segundo a metodologia de Tedesco et al. (1995), substâncias de reserva (folhas e hastes), segundo método descrito por Priestley (1965), colonização radicular (através da relação: número de segmentos infectados/total analisado) e avaliação da presença e intensidade de hifas (índices: 0 - ausência de estruturas; 1 - presença fraca; 2 - moderada; 3 - intensa), vesículas e arbúsculos (índices: 0 - ausência de estruturas; 1 - 1 a 50 estruturas; 2 - 51 a 100 estruturas; 3 - mais de 100 estruturas por centímetro de radicela), segundo metodologia descrita por Nemec (1992).

Os dados foram submetidos à análise de variância executada pelo programa SAS e as médias foram comparadas pelo teste de Duncan, ao nível de 5\% de significância.

\section{RESULTADOS E DISCUSSÃO}

A inoculação com Glomus etunicatum proporcionou os maiores incrementos em altura, diâmetro, área foliar, biomassa fresca e seca dos tecidos da parte aérea, enquanto Glomus clarum induziu um comportamento intermediário de todos os parâmetros de crescimento (Tabela 1). Gigaspora margarita foi ineficiente, apresentando comportamento semelhante às plantas-testemunhas para todos os parâmetros de desenvolvimento, exceção feita à biomassa seca, que foi superior à testemunha, mas inferior às outras espécies de FMA (Tabela 1).

Esses resultados são similares aos obtidos por Chu (1999), que, ao inocular as espécies Scutellospora gilmorei e Gigaspora margarita isolados de pomares de açaí, separadamente, em plantas de açaizeiro, observou aumentos de $92 \%$ de altura e $116 \%$ de diâmetro nas plantas inoculadas com Scutellospora gilmorei, enquanto as inoculadas com Gigaspora margarita apresentaram crescimento em altura e diâmetro semelhantes às testemunhas. Segundo Aguiar et al. (2004), os FMA nativos de determinado ambiente podem ser mais adaptados às condições prevalecentes nesse ambiente, variando a resposta em função da compatibilidade da simbiose planta - FMA. 
Tabela 1 - Altura, diâmetro, área foliar, biomassa fresca e seca da parte aérea (folhas e hastes) de plantas do portaenxerto de pessegueiro 'Aldrighi', inoculado com três espécies de FMA e sem inoculação. Eldorado do Sul, RS, 2006.

\begin{tabular}{lccccc}
\hline \multirow{2}{*}{ Tratamento } & \multirow{2}{*}{$\begin{array}{c}\text { Altura* } \\
(\mathrm{cm})\end{array}$} & \multirow{2}{*}{$\begin{array}{c}\text { Diâmetro } \\
(\mathrm{mm})\end{array}$} & \multirow{2}{*}{$\begin{array}{c}\text { Área foliar } \\
\left(\mathrm{cm}^{2} / \text { planta }\right)\end{array}$} & \multicolumn{2}{c}{ Biomassa da parte aérea } \\
\cline { 6 - 7 } & & & & Fresca $(\mathrm{g})$ & Seca $(\mathrm{g})$ \\
\hline Gigaspora margarita & $132,00 \mathrm{c}$ & $6,20 \mathrm{c}$ & $457,02 \mathrm{c}$ & $106,00 \mathrm{c}$ & $45,00 \mathrm{c}$ \\
Glomus clarum & $146,53 \mathrm{~b}$ & $6,79 \mathrm{~b}$ & $613,01 \mathrm{~b}$ & $120,00 \mathrm{~b}$ & $51,00 \mathrm{~b}$ \\
Glomus etunicatum & $171,00 \mathrm{a}$ & $7,30 \mathrm{a}$ & $796,90 \mathrm{a}$ & $139,00 \mathrm{a}$ & $62,00 \mathrm{a}$ \\
Testemunha & $130,00 \mathrm{c}$ & $6,35 \mathrm{c}$ & $450,25 \mathrm{c}$ & $108,00 \mathrm{c}$ & $37,00 \mathrm{~d}$ \\
\hline C.V. $(\%)$ & 7,98 & 7,77 & 7,58 & 3,44 & 2,77 \\
\hline
\end{tabular}

*Médias seguidas pela mesma letra minúscula na coluna, não diferem entre si pelo teste de Duncan, ao nível de 5\% de significância.

A área foliar é um importante parâmetro, por definir a taxa de fotossíntese realizada na planta, que resulta na maior ou menor produção de fotoassimilados que serão, em parte, translocados para os FMA (CAVALCANTE et al., 2002). Silva et al. (2004), afirmam que o aumento da taxa fotossintética de plantas inoculadas com FMA está diretamente relacionado ao aumento da área foliar, o que proporciona aumento do crescimento vegetativo e acúmulo de biomassa fresca e seca. Segundo Silveira et al. (2002), plantas colonizadas por FMA que apresentem área foliar semelhante ou até mesmo inferior às plantas não colonizadas, podem indicar uma ineficiência do micélio externo do fungo em absorver e translocar água e nutrientes para a planta, ao mesmo tempo em que a planta apresenta gasto energético com o FMA, caracterizando, dessa forma, não uma relação simbiótica, mas, sim, parasítica, o que pode ter ocorrido nas plantas inoculadas com Gigaspora margarita.

Cavalcante et al. (2002) e Costa et al. (2005), que trabalharam com maracujazeiro-amarelo e mangabeira, respectivamente, também observaram que a simbiose planta-FMA aumentou a área foliar das frutíferas inoculadas em relação à testemunha. Por outro lado, Silva et al. (2004), trabalhando com mudas de maracujazeiro-doce inoculadas com quatro espécies de FMA isolados de pomares de maracujazeiros-doces, afirmam que mudas inoculadas com Glomus etunicatum apresentaram crescimento lento e área foliar semelhante às plantas-testemunhas, enquanto plantas inoculadas com espécies do gênero Gigaspora apresentaram os maiores resultados em termos de crescimento, biomassa e área foliar. Tais relatos não coincidem com os resultados obtidos nesse trabalho, uma vez que o isolado nativo da espécie Glomus etunicatum foi o que apresentou os maiores resultados em termos de desenvolvimento vegetativo, ao mesmo tempo em que Gigaspora margarita apresentou desempenho semelhante às plantas-testemunhas.
Os teores de nitrogênio, fósforo e potássio encontrados na parte aérea (folhas e hastes) de plantas inoculadas com Glomus clarum foram inferiores aos das inoculadas com Glomus etunicatum, mas superiores às inoculadas com Gigaspora margarita e a testemunha, os quais foram iguais entre si (Tabela 2). Para o cálcio e o magnésio, todos os tratamentos com FMA foram iguais entre si e inferiores à testemunha.

Com relação às substâncias de reserva, verifica-se que plantas inoculadas com Glomus etunicatum apresentaram os maiores porcentuais, enquanto que as inoculadas com Glomus clarum apresentaram comportamento intermediário, superior ao das inoculadas com Gigaspora margarita e das plantas-testemunhas, que foram iguais entre si (Tabela 2).

Segundo Scatena \& Scremin-Dias (2003), plantas mais altas e com maior área foliar apresentam maior capacidade de fotossíntese e produção de fotoassimilados, proporcionando acúmulo de biomassa e, por conseguinte, maior nível de carbono assimilado, o que permite um fluxo mais intenso de carboidratos no sentido radicial onde uma parte seria utilizada pelos FMA na sua nutrição e acumulação em estruturas de reserva, e o restante seria acumulado nos tecidos de armazenamento da planta, na forma de substâncias de reserva.

Os maiores porcentuais de nitrogênio nos tecidos vegetais das plantas inoculadas com Glomus clarum e Glomus etunicatum contribuiu para o maior crescimento em altura, diâmetro, área foliar, biomassa fresca e seca da parte aérea, quando comparados a Gigaspora margarita e a testemunha (Tabelas 1 e 2). Porém, somente o tratamento com Glomus etunicatum apresentou teor de nitrogênio dentro da normalidade, entre 3,26 a 4,53\% de nitrogênio na planta (COMISSÃO DE QUÍMICA E FERTILIDADE DO SOLO - RS e SC, 2004). No caso de Glomus clarum, conforme observado por Agostini (2002), o maior crescimento das plantas pode ter proporcionado 
Tabela 2 - Teores de macronutrientes e substâncias de reserva encontrados na parte aérea (folhas e hastes) de plantas do porta-enxerto de pessegueiro 'Aldrighi', inoculado com três espécies de FMA e sem inoculação. Eldorado do Sul, RS, 2006.

\begin{tabular}{|c|c|c|c|c|c|}
\hline \multirow{2}{*}{ Tratamento } & \multicolumn{5}{|c|}{ Macronutrientes na parte aérea $(\%)$} \\
\hline & $\mathrm{N}^{*}$ & $\mathrm{P}$ & $\mathrm{K}$ & $\mathrm{Ca}$ & $\mathrm{Mg}$ \\
\hline Gigaspora margarita & $2,39 \mathrm{c}$ & $0,14 \mathrm{c}$ & $2,14 \mathrm{c}$ & $1,17 b$ & $0,40 \mathrm{~b}$ \\
\hline Glomus clarum & $3,12 b$ & $0,19 b$ & $2,28 b$ & $1,18 b$ & $0,41 b$ \\
\hline Glomus etunicatum & $3,36 \mathrm{a}$ & $0,24 \mathrm{a}$ & $2,50 \mathrm{a}$ & $1,18 b$ & $0,40 \mathrm{~b}$ \\
\hline Testemunha & $2,38 \mathrm{c}$ & $0,14 \mathrm{c}$ & $2,13 \mathrm{c}$ & $1,75 \mathrm{a}$ & $0,59 a$ \\
\hline \multirow[t]{2}{*}{ C.V.(\%) } & 7,61 & 6,33 & 9,64 & 12,44 & 5,36 \\
\hline & \multicolumn{5}{|c|}{ Substâncias de reserva da parte aérea (\%) } \\
\hline Gigaspora margarita & \multicolumn{5}{|c|}{$16,61 c$} \\
\hline Glomus clarum & \multicolumn{5}{|c|}{$22,36 b$} \\
\hline Glomus etunicatum & \multicolumn{5}{|c|}{$29,09 a$} \\
\hline Testemunha & \multicolumn{5}{|c|}{$16,59 \mathrm{c}$} \\
\hline C.V.(\%) & \multicolumn{5}{|c|}{8,79} \\
\hline
\end{tabular}

*Médias seguidas pela mesma letra, na coluna, não diferem entre si pelo teste de Duncan, ao nível de 5\% de significância.

um efeito de diluição do teor de nitrogênio nos tecidos vegetais.

Segundo Tedesco et al. (1995), o fósforo é o elemento que apresenta grande mobilidade no tecido vegetal, porém é pouco móvel no solo, além de apresentar baixa solubilidade. Dessa forma, ao propiciarem maior absorção desse elemento, os FMA assumem papel fundamental para os vegetais (MINHONI \& AULER, 2003).

Percebeu-se uma elevação gradual do $\mathrm{pH}$ do substrato ao longo do experimento (Tabela 3). Isso favoreceu a colonização das raízes pelas espécies Glomus clarum e Glomus etunicatum e proporcionou maior absorção de fósforo do substrato, já que essas espécies predominam em solo pouco ácido ou neutro. Porém, no caso de Gigaspora margarita, o pH inicial do substrato era demasiadamente elevado para proporcionar resposta dos esporos e permitir uma colonização elevada das raízes. Isto porque, segundo Silveira et al. (2002), esta espécie tem predileção por acidez elevada. Além disto, a elevação gradual do $\mathrm{pH}$ dificultou ainda mais o estabelecimento e o desenvolvimento da simbiose planta - Gigaspora margarita, o que se observa pelo porcentual de fósforo nas plantas inoculadas com essa espécie, semelhante às plantas-testemunhas (Tabela 2).

Os porcentuais de matéria orgânica e os teores de fósforo, potássio, cálcio e magnésio sofreram redução desde a implantação até a avaliação do experimento (Tabela 3). $\mathrm{O}$ pH e os teores de cálcio e magnésio não diferiram entre os diferentes tratamentos após 11 meses de cultivo Já, os percentuais de matéria orgânica e os teores de fósforo e cálcio foram significativamente menores nos substratos onde as plantas foram inoculadas com Glomus etunicatum. Nos substratos onde as plantas foram cultivadas com Gigaspora margarita, os porcentuais de matéria orgânica e os teores de fósforo e potássio foram superiores aos demais FMA e semelhantes ao das plantastestemunha. Nos substratos daquelas inoculadas com Glomus clarum, os porcentuais de matéria orgânica e os teores de fósforo e potássio apresentaram teores intermediários (Tabela 3).

Conforme relato por Minhoni \& Auler (2003) e Silveira et al. (2002), algumas espécies do gênero Gigaspora apresentam comportamento parasítico quando em substrato com elevado porcentual de matéria orgânica (Tabela 3), confirmando os resultados desse estudo.

Por outro lado, segundo Medeiros \& Raseira (1998), o porcentual de fósforo contido no substrato é considerado baixo para a cultura do pessegueiro (entre 4,1-9,0 $\mathrm{mg} \mathrm{dm}^{-3}$ ) (Tabela 3). O fato de que os substratos utilizados para plantas inoculadas com Glomus clarum e Glomus etunicatum apresentaram níveis de fósforo inferiores ao das testemunhas e de Gigaspora margarita, mostra que essas espécies foram eficientes em promover maior absorção desse nutriente, confirmado pelos teores nos tecidos (Tabela 2). Por sua vez, o substrato utilizado para o cultivo das plantas inoculadas com Gigaspora margarita não mostrou diferenças em relação à testemunha, ou seja, essa espécie não proporcionou aumento de absorção desse elemento. 
Tabela 3 - Matéria orgânica (MO), pH e macronutrientes encontrados nos substratos no momento da implantação do experimento e aos 330 dias após o plantio com o porta-enxerto de pessegueiro 'Aldrighi', inoculado com três espécies de FMA e sem inoculação. Eldorado do Sul, RS, 2006.

\begin{tabular}{|c|c|c|c|c|c|c|}
\hline \multirow{4}{*}{ Tratamento } & $\mathrm{pH}^{*}$ & $\begin{array}{l}\text { MO } \\
(\%)\end{array}$ & $\begin{array}{c}\mathrm{P} \\
\left(\mathrm{mg} \cdot \mathrm{dm}^{-3}\right)\end{array}$ & $\begin{array}{c}\mathrm{K} \\
\left(\mathrm{mg} \cdot \mathrm{dm}^{-3}\right)\end{array}$ & $\begin{array}{c}\mathrm{Ca} \\
\left(\mathrm{cmol}_{\mathrm{c}} \cdot \mathrm{L}^{-1}\right)\end{array}$ & $\begin{array}{c}\mathrm{Mg} \\
\left(\mathrm{cmol}_{\mathrm{c}} \cdot \mathrm{L}^{-1}\right)\end{array}$ \\
\hline & \multicolumn{6}{|c|}{ Implantação do experimento - 02/09/2005 } \\
\hline & 5,2 & 4,8 & 7,9 & 59 & 4,2 & 2,2 \\
\hline & \multicolumn{6}{|c|}{ Muda pronta para enxertia - 21/08/2006 } \\
\hline Gigaspora margarita & 6,0 & $4,2 \mathrm{a}$ & $6,4 a$ & $40 \mathrm{a}$ & 3,0 & 1,1 \\
\hline Glomus clarum & 6,0 & $3,9 b$ & $5,0 \mathrm{~b}$ & $32 b$ & 3,0 & 1,1 \\
\hline Glomus etunicatum & 6,0 & $3,5 \mathrm{c}$ & $4,1 \mathrm{c}$ & $25 c$ & 2,9 & 1,1 \\
\hline Testemunha & 5,9 & $4,2 \mathrm{a}$ & $6,6 \mathrm{a}$ & $42 \mathrm{a}$ & 3,0 & 1,2 \\
\hline
\end{tabular}

*Médias seguidas pela mesma letra, na coluna, não diferem entre si pelo teste de Duncan, ao nível de 5\% de significância.

Assim como no caso do fósforo, o potássio possui boa mobilidade na planta, mas no solo ele é transportado lentamente até as raízes, por difusão (TEDESCO et al., 1995). Além disso, por ser o potássio um elemento importante na planta, ao participar de compostos orgânicos das células, atuando no equilíbrio elétrico das mesmas, ativando enzimas e participando da regulação da abertura e fechamento dos estômatos, interferindo, assim, na fotossíntese, uma maior absorção desse elemento promovida pelos FMA é benéfica para as plantas (TAIZ \& ZIEGER, 2004). No caso de plantas inoculadas com Glomus clarum e Glomus etunicatum essa tendência mostrou-se superior ao daquelas inoculadas com Gigaspora margarita e nas não inoculadas (Tabelas 2 e 3).

Silveira et al. (2002), afirmam que o cálcio e o magnésio são vitais para o desenvolvimento das plantas, por participarem da regulação da hidratação e da ativação de enzimas, além de participarem da fotossíntese, no caso do magnésio. Porém, segundo relato de Souza (2000), os FMA têm a capacidade de reduzir a absorção desses elementos, em virtude de um efeito tampão proporcionado pelos fungos, que diminui a absorção desses elementos pelas raízes das plantas, o que está em concordância com os resultados obtidos neste trabalho (Tabela 2).

Silveira et al. (2002) relatam que plantas de portaenxerto de abacateiro, inoculadas com Gigaspora margarita, apresentaram os níveis mais baixos de cálcio e magnésio nos tecidos das plantas, o que indica uma inibição na absorção desses elementos, o que concorda com os resultados obtidos nesse trabalho (Tabela 2).

A eficiência dos FMA correlacionou-se com a colonização radicial, pois plantas inoculadas com Glomus clarum e Glomus etunicatum apresentaram altas taxas de colonização, 77 e $92 \%$ respectivamente, enquanto que Gigaspora margarita apresentou colonização considerada baixa, de 30,33\% (Tabela 4). Além disso, o tratamento com Glomus etunicatum foi o que apresentou o maior número de esporos recuperados no substrato, enquanto que Glomus clarum foi superior a Gigaspora margarita. $\mathrm{Na}$ amostra retirada da testemunha não foram encontrados esporos (Tabela 4).

As plantas inoculadas com Glomus clarum e Glomus etunicatum apresentaram índices de colonização com hifas, vesículas e arbúsculos considerados medianos (NEMEC, 1992), sendo que Glomus etunicatum apresentou os maiores resultados, enquanto Glomus clarum apresentou comportamento intermediário. Gigaspora margarita apresentou índices de hifas e arbúsculos considerados baixos, sem vesículas (NEMEC, 1992). Cabe salientar, com relação às vesículas, que os fungos do gênero Gigaspora não formam vesículas intrarradiciais, mas sim células auxiliares extra-radiculares.

Conforme relatado por Mazzoni-Viveiros \& Trufem (2004), um maior diâmetro de caule, que seria proporcionado pelos FMA, permitiria um aumento do fluxo ascendente de água e de nutrientes, e de seiva elaborada, no sentido descendente. Na prática, isso significa antecipar a enxertia, acelerando a produção da muda. Tal relato coincide com os resultados obtidos com Glomus clarum e Glomus etunicatum, uma vez que as mesmas apresentaram os maiores resultados em termos de altura, diâmetro e área foliar, apresentando, também, quantidades superiores de substâncias de reserva, enquanto que Gigaspora margarita apresentou crescimento vegetativo, área foliar e substâncias de reserva semelhantes à testemunha. 
Tabela 4 - Colonização radicial, número de esporos presentes no substrato e presença de hifas, vesículas e arbúsculos encontrados em raízes de plantas do porta-enxerto de pessegueiro 'Aldrighi', inoculadas com três espécies de FMA e sem inoculação. Eldorado do Sul, RS, 2006.

\begin{tabular}{lccccc}
\hline \multirow{2}{*}{ Tratamentos } & Colonização $(\%)^{*}$ & \begin{tabular}{c} 
Esporos $\left(\mathrm{n}^{\circ}\right.$ \\
médio/100g \\
\cline { 5 - 6 }
\end{tabular} & & \multicolumn{2}{c}{ Presença de estruturas de FMA } \\
\cline { 5 - 7 } & solo seco) & & Hifas & Vesículas & Arbúsculos \\
\hline Gigaspora margarita & $30,33 \mathrm{c}$ & $59,00 \mathrm{c}$ & $0,78 \mathrm{c}$ & $0,00 \mathrm{c}$ & $0,53 \mathrm{c}$ \\
Glomus clarum & $77,00 \mathrm{~b}$ & $160,00 \mathrm{~b}$ & $1,44 \mathrm{~b}$ & $0,70 \mathrm{~b}$ & $0,84 \mathrm{~b}$ \\
Testemus etunicatum & $92,00 \mathrm{a}$ & $314,00 \mathrm{a}$ & $1,57 \mathrm{a}$ & $1,36 \mathrm{a}$ & $1,50 \mathrm{a}$ \\
\hline C.V. $(\%)$ & $0,00 \mathrm{~d}$ & $0,00 \mathrm{~d}$ & $0,00 \mathrm{~d}$ & $0,00 \mathrm{c}$ & $0,00 \mathrm{~d}$ \\
\hline
\end{tabular}

*Médias seguidas pela mesma letra, na coluna, não diferem entre si pelo teste de Duncan, ao nível de 5\% de significância.

Outro fato a ser destacado é que, conforme observado por Silva et al. (2004), quando os FMA são utilizados na produção de mudas frutíferas em condições homogêneas de substrato e de ambiente, os benefícios proporcionados pela simbiose podem ser atribuídos à combinação FMA X hospedeiro, devido à existência de uma maior compatibilidade funcional entre a frutífera e a espécie de FMA. Dessa forma, segundo afirmam Costa et al. (2005), o crescimento das plantas frutíferas inoculadas pode ser favorecido pela associação simbiótica, desde que seja aplicado à espécie de FMA mais compatível com o genótipo da frutífera.

\section{CONCLUSÕES}

O uso de fungos micorrízicos arbusculares isolados de pomares de pessegueiro acelera o desenvolvimento vegetativo e melhora o estado nutricional do porta-enxerto de pessegueiro 'Aldrighi'.

A eficiência da simbiose é variável com a espécie de FMA, em que Glomus etunicatum sobressai-se, seguido por Glomus clarum, enquanto Gigaspora margarita mostra-se ineficiente em incrementar o desenvolvimento das plantas do porta-enxerto de pessegueiro 'Aldrighi'.

\section{REFERÊNCIAS BIBLIOGRÁFICAS}

AGOSTINI, S. Influência de Fungos Micorrízicos Arbusculares sobre o desenvolvimento vegetativo de portaenxertos de videira. 2002. 63 f. Dissertação (Mestrado) Universidade Federal do Rio Grande do Sul, Porto Alegre, 2002.

AGUIAR, R. L. F.; MAIA, L. C.; SALCEDO, I. N.; SAMPAIO, E. V. S. B. Interação entre fungos micorrízicos arbusculares e fósforo no desenvolvimento da Algaroba [Prosopis juliflora (SW) DC]. Revista Árvore, Viçosa, v. 28, n. 4, p. 589-598, 2004.
CAVALCANTE, U. M. T.; MAIA, L. C.; MELO, A. M. M.; SANTOS, V. F. Influência da densidade de fungos micorrízicos arbusculares na produção de mudas de maracujazeiro-amarelo. Pesquisa Agropecuária Brasileira, Brasília, v. 37, n. 5, p. 634-649, 2002.

CHU, E. Y. The effects of arbuscular mycorrhizal fungi inoculation on Euterpe oleracea Mart. Seedlings. Pesquisa Agropecuária Brasileira, Brasília, v. 34, n. 6, p. 1019-1024, 1999.

COMISSÃO DE QUÍMICA E FERTILIDADE DO SOLO, RS e SC. Manual de adubação e calagem para o Rio Grande do Sul e Santa Catarina. Porto Alegre: SBCS, 2004. 394 p.

COSTA, C. M. C.; CAVALCANTE, U. M. T.; GOTO, B. T.; SANTOS, V. F.; MAIA, L. C. Fungos micorrízicos arbusculares e adubação fosfatada em mudas de mangabeira. Pesquisa Agropecuária Brasileira, Brasília, v. 40, n. 30, p. 225-232, 2005.

DODD, J. C. The role of arbuscular mycorrizal fungi in agro: and natural ecosystems. Outlook on Agriculture, London, v. 29, n. 1, p. 55-62, 2000.

EMPRESA BRASILEIRA DE PESQUISA AGROPECUÁRIA. Centro Nacional de Pesquisa de Solos. Sistema brasileiro de classificação de solos. Brasília, DF, 1999. 412 p.

GEDERMANN, J. W.; NICOLSON, T. H. Spores of mycorrhizal Endogone extracted from soil by wet sieving and decantinf. Transactions of the British Mycological Society, London, v. 46, p. 235-244, 1963.

JENKINS, W. R. A rapid centrifugal-flotation technique for separating nematodes from soil. Plant Diseases Report, Saint Paul, v. 48, p. 691-692, 1964. 
MAZZONI-VIVEIROS, S. C.; TRUFEM, S. F. B. Efeitos da poluição aérea e edáfica no sistema radicular de Tibouchina pulchra Cogn. (Melastomataceae) em área de mata Atlântica: associações micorrízicas e morfologia. Revista Brasileira de Botânica, São Paulo, v. 27, n. 2, p. 337-348, 2004.

MEDEIROS, C. A. B.; RASEIRA, M. do C. B. A cultura do pessegueiro. Brasília, DF: Embrapa-SPI, 1998. 350 p.

MINHONI, M. T. A.; AULER, P. A. M. Efeito do fósforo, fumigação do substrato e fungo micorrízico arbuscular sobre o crescimento de plantas de mamoeiro. Revista Brasileira de Ciência do Solo, Campinas, v. 27, p. 841-847, 2003.

NEMEC, S. Glomus intraradix effects on citrus roostock seedling growth in various potting media. Journal of Agricultural Science, Cambridge, v. 118, p. 315-323, 1992.

PRIESTLEY, G. A. New method for the estimation of the resources of apple tress. Journal of the Science of Food and Agriculture, London, v. 16, p. 717-721, 1965.

RILLING, M. C.; TRESEDER, K. K.; ALLEN, N. F. Global change and mycorrhizal fungi. In: HEIJDERN, M. van der; SANDERS, I. (Eds.). Mycorrhizal ecology. New York: Springer, 2002. p. 135-160.

SCATENA, V. L.; SCREMIN-DIAS, E. Parênquima, Colênquima e Esclerênquima. In: APPEZZATO-DA-
GLÓRIA, B.; CARMELLO-GUERREIRO, S. M. (Eds.). Anatomia vegetal. Viçosa: UFV, 2003. p. 109-127.

SCHENCK, N. C.; PEREZ, Y. Manual for the identification of VA mycorrhizal fungi. Gainesville: University of Florida, 1988. $240 \mathrm{p}$.

SILVA, M. A.; CAVALCANTE, U. M. T.; SILVA, F. S. B.; SOARES, S. A. G.; MAIA, L. C. Crescimento de mudas de maracujazeiro-doce (Passiflora alata Curtis) associadas a fungos micorrízicos arbusculares (Glomeromycota). Acta Botanica Brasílica, Manaus, v. 18, n. 4, p. 981-985, 2004.

SILVEIRA, S. V.; SOUZA, P. V. D.; KOLLER, O. C. Inflência de fungos micorrízicos arbusculares sobre o desenvolvimento vegetativo de porta-enxertos de abacateiro. Pesquisa Agropecuária Brasileira, Brasília, v. 37, n. 3, p. 303-309, 2002.

SOUZA, P. V. D. de. Interação entre micorrizas arbusculares e ácido giberélico no desenvolvimento vegetativo de plantas de Citrange Carrizo. Ciência Rural, Santa Maria, v. 30, n. 5 , p. $783-787,2000$.

TAIZ, L.; ZEIGER, E. Fisiologia vegetal. 3. ed. Porto Alegre: Artmed, 2004. 717 p.

TEDESCO, M. J.; GIANELLO, C.; BISSANI, C. A.; BOHNEN, H.; VOLKWEISS, S. J. Análises de solo, plantas e outros materiais. 2. ed. Porto Alegre: UFRGS, 1995. 174 p. (Boletim técnico, 5). 